\title{
$\angle S$ Research Square \\ Identification of cell cycle dependent methotrexate resistance in placenta site trophoblastic tumors
}

\section{Jing Xu}

Obstetrics and Gynecology Hospital of Fudan University

\section{Ling Zhang}

Obstetrics and Gynecology Hospital of Fudan University

\section{Qiyu Liu}

Obstetrics and Gynecology Hospital of Fudan University

\section{Luyao Ren}

Fudan University

\section{Ke Li}

Obstetrics and Gynecology Hospital of Fudan University

\section{Baozhu Cai}

Fudan University

\section{Yunke Huang}

Obstetrics and Gynecology Hospital of Fudan University Jing Wang

Obstetrics and Gynecology Hospital of Fudan University

\section{Qin Li}

Obstetrics and Gynecology Hospital of Fudan University

Yu Kang ( $\square$ yukang@fudan.edu.cn )

Obstetrics and Gynecology Hospital of Fudan University

\section{Congjian Xu}

Obstetrics and Gynecology Hospital of Fudan University

\section{Research}

Keywords:

Posted Date: April 28th, 2020

DOI: https://doi.org/10.21203/rs.3.rs-24085/v1

License: (c) (1) This work is licensed under a Creative Commons Attribution 4.0 International License. Read Full License 


\section{Abstract}

Background The purpose is to study the mechanism of chemotherapy resistance in Placental site trophoblastic tumor(PSTT).

Methods We established PSTT cell lines by primary culture of a surgically resected PSTT tissues and identified the expression of immune-phenotype markers(HLA-G, $\beta$-catenin, CD146, Muc4, hPL, hCG) by immunofluorescence. We measured the IC50 value of methotrexate(MTX), etoposide(VP-16), actinomycin-D(Act-D), cisplatin(DDP), fluorouracil(5-FU) and paclitaxel(TAX) in PSTTs and used a special Mini patient-derived xenograft (Mini PDX) model to evaluate effectiveness of these drugs in vivo. Given that MTX is a cell cycle-dependent chemotherapeutic, we analyzed cell cycle characteristics of PSTT and choriocarcinoma cell lines by flow cytometry and then analyzed RNA profiles and WGS data of the PSTT cell lines to identify the potential mechanism.

Results We identified the expression of HLA-G, $\beta$-catenin, CD146, hPL and hCG in PSTT cell lines. The IC50 value of MTX was $4.922 \mathrm{mg} / \mathrm{ml}$ in PSTT-1, $4.525 \mathrm{mg} / \mathrm{ml}$ in PSTT-2, $5.117 \mathrm{mg} / \mathrm{ml}$ in PSTT-3, $0.0166 \mu \mathrm{g} / \mathrm{ml}$ in JEG-3 cells (pष0.001), and $0.01 \mu \mathrm{g} / \mathrm{ml}$ in JAR cells ( $\mathrm{p} \otimes 0.001$ ), with nearly 50,000 -fold increase in PSTTs than in choriocarcinoma, indicating that PSTTs are resistant to MTX in vitro. The Mini PDX model revealed that PSTTs are also resistant to MTX in vivo. Cell cycle analysis showed dysregulation of G1/S transition and cell cycle arrest in PSTT cell lines. RNA sequencing profile also identified cell cycle-associated genes which were differentially expressed in PSTT cells than in choriocarcinoma cell.

Conclusions We found PSTTs are resistant to MTX in vitro and in vivo compared to choriocarcinoma. Mechanisms could be focused on dysregulation of the G1/S transition and cell cycle arrest.

\section{Background}

PSTT, a rare type of gestational trophoblastic neoplasia(GTN), is biologically unique from choriocarcinoma because of its relatively slower growth rate, lower serum hCG levels, later onset of metastatic potential and greater resistance to chemotherapy ${ }^{(1)}$. PSTTs typically occur in females of reproductive age, arises months to years after a term gestation, and can develop after any pregnancy ${ }^{(1-4)}$. Most GTNs are exquisitely susceptible to chemotherapy, while PSTTs are relatively chemoresistance $e^{(3,5)}$. Therefore, hysterectomy combined with chemotherapy has been considered the classic treatment for PSTT, and hysterectomy is suggested to be performed immediately after PSTT diagnosis ${ }^{(6,7)}$. However, hysterectomy may be unacceptable for the patients who desire to preserve their fertility. Fertility preservation could be possible in theory if we had effective chemotherapy for PSTT so it's important to understand the mechanism of chemoresistance in PSTT.

The preferential first-line treatment for PSTT is EP/EMA or EMA/EP based on the NCCN Clinical Practice Guidelines. Once PSTT patients are resistant to the EMA/EP regimen, no effective options remain due to 
the unclear chemoresistance mechanism. Therefore, the mortality rate of PSTT is approximately $10 \%$ to $20 \%$ according to published reports and a poor prognosis for patients with multiple metastases, especially since death often occurs within one year of diagnosis ${ }^{(8,9)}$. The 10 -year survival rate of patients with PSTT is $49 \%$, and the 5 -year survival rate of relapsed patients is less than $30 \%(3,10)$. Therefore, it is important to solve the resistance problem and increase the survival rate. However, basic scientific research on PSTT is rare, and thus, the molecular mechanism of chemotherapy resistance is unclear.

Methotrexate(MTX) is a common chemotherapy drug in clinical practice that produces the first remission in leukemia and the first cure of choriocarcinoma ${ }^{(11)}$. MTX is the single most commonly used agent in choriocarcinoma patients without metastasis or with a low risk of metastasis ${ }^{(12,13)}$, but approximately $30 \%$ of patients develop resistance to MTX monotherapy ${ }^{(14)}$. Although MTX plays an important role in the treatment of low-risk choriocarcinoma, there are no studies on the effect of MTX in PSTT thus far.

MTX, a folate antagonist, is traditionally regarded as a proliferation inhibitor that blocks the $S$ phase of the cell cycle ${ }^{(15)}$. Therefore, the normal cell cycle plays an important role in MTX pharmacology. Many studies have verified that cell cycle disorders are related to MTX resistance in some solid tumors ${ }^{(15-17)}$.

In our study, we established three PSTT cell lines to study the underlying molecular mechanisms. We found that PSTT is significantly resistant to MTX in vitro and in vivo. Therefore, we studied the cell cycleassociated MTX resistance mechanism and proposed possible functional gene mutations.

\section{Materials And Methods}

\section{Establishment of PSTT-1, PSTT-2 and PSTT-3.}

This study was approved by the ethics committee (IRB number:2016-56). After the consent process, fresh tissue, examined by a pathologist, was derived by hysterectomy from three PSTT patients who were admitted to the Obstetrics and Gynecology Hospital of Fudan University. The $1 \mathrm{~cm}^{3}$ tissues were immersed in sterile PBS(Gibco, USA) to remove connective tissue and necrotic tissue. The tissues were cut into pieces of approximately $1 \mathrm{~mm}^{3}$ and inoculated into T25 flasks. Half of the medium was replaced every 3 days. After culture for approximately 7 weeks, the cells were passaged for the first time by digestion with $0.25 \%$ trypsin(Gibco, USA). The cell suspension in RPMI-1640 medium(Gibco, USA) was placed into a culture flask (containing serum-free medium), and removed some of the cells which were attached after 10-15 minutes. The above steps were repeated 3 to 5 times to completely separate the tumor cells from the fibroblasts. The routine in vitro culture conditions involved RPMI-1640 cell culture medium containing 10\% FBS(Sigma, USA), 1\% sodium pyruvate(Gibco, USA), 1\% L-glutamine(Gibco, SA), $1 \%$ HEPES(Gibco, USA $1 \%$ antibody(Gibco, USA), and placed at $37^{\circ} \mathrm{C}$ incubator with $5 \% \mathrm{CO} 2$.

\section{Immunofluorescence:}


Tumor cells( $2 \times 10^{4}$ cells, 200 ul $)$ were cytospin onto a slide, fixed with $4 \%$ paraformaldehyde and permeabilized with $0.3 \%$ Triton X-100, and then blocked with $5 \%$ goat serum. Then cells were incubated at $4{ }^{\circ} \mathrm{C}$ overnight with primary monoclonal antibodies: $\operatorname{HLA}-\mathrm{G}(1: 50, \mathrm{ab} 52455, \mathrm{Abcam}, \mathrm{USA}), \beta-$ catenin(1:200,sc-133238,Santa Cruz Biotechnology, USA), CD146(1:400, GTX108777, GeneTex, USA), HPL(1:200, ab15554,Abcam, USA), and HCG(1:200, ab9376, Abcam, USA). Subsequently, the cells were probed with anti-mouse or goat-anti-rabbit secondary antibody. Finally, the cells were mounted with DAPIcontaining mounting medium(S36973,Thermo Fisher, MA, USA). Images were captured with a fluorescence microscope(Leica,Germany) with Leica Application Suite V4 software and edited with Photoshop(Adobe, USA).

\section{Cell growth curvel}

Cells in the logarithmic growth phase were adjusted to a density of $2 \times 10^{4}$ cells $/ \mathrm{mL}$ after trypsinization. After mixing, the cells were seeded in triplicate in a 96-well plate. After 6 days of culture, CCK-8 reagent(Dojindo, Kumamoto, JPN) was added for $4 \mathrm{~h}$, and the absorbance(D) at $450 \mathrm{~nm}$ was measured by a microplate reader.

\section{Chromosomal karyotype analysis:}

Cells in Logarithmic growth phase were treated with $0.25 \mu \mathrm{g} / \mathrm{mL}$ colchicine for $6 \mathrm{~h}$ at $37^{\circ} \mathrm{C}$ overnight. The metaphase cells were harvested and fixed with methanol-glacial acetic acid(3:1). The specimens were trypsinized, stained with Giemsa stain, and counted under a microscope. The karyotype analysis was performed on the split phase with good dispersion and moderate staining.

\section{Cell proliferation assay:}

The $50 \%$ inhibitory concentration $50 \%$ was determined with a CCK-8 (Dojindo, Kumamoto, JPN) assay as per the protocols. A total of $5 \times 10^{3} \mathrm{JAR}$, JEG-3, or PSTT cells were seeded into 96-well plates at approximately $50 \%$ confluence. Then, the cells were treated with drugs for $48-96 \mathrm{~h}$, and $10 \mu \mathrm{l}$ of CCK-8 was added to each well for $2 \mathrm{~h}$ at $37^{\circ} \mathrm{C}$. The absorbance was measured at $450 \mathrm{~nm}$ with a microplate reader (BioTek, Winooski, USA). Resistance Index $(\mathrm{RI})=$ IC50 of drug-resistant cells / IC50 of parental cells.

\section{Establishing the MiniPDX model:}

We developed an in vivo drug sensitivity MiniPDX assay using a modified microencapsulation and hollow fiber culture system (OncoVee MiniPDX®, LIDE Biotech) according to the manufacturer's instruction.

Tumors $\geq 500 \mathrm{~mm}^{3}$ with less than $30 \%$ necrosis were used. Briefly, tumor tissues were washed with HBSS to remove non-tumor tissues and necrotic tumor tissue, and then were digested with collagenase at $37^{\circ} \mathrm{C}$ for 1-4 h. Cells were pelleted by centrifugation at $600 \mathrm{~g}$ for $5 \mathrm{~min}$ and then were removed of blood cells and fibroblasts with magnetic beads. Cells were then washed with HBSS and filled into OncoVee ${ }^{\circledR}$ capsules, which were made of hollow fiber membrane with a $500 \mathrm{kD}$ pore size allowing small molecules 
less than $500 \mathrm{kD}$ to move in and out freely while keeping cells within the capsule ${ }^{(18-21)}$. Capsules were implanted subcutaneously via a small skin incision with 3 capsules per mouse (5-week-old nu/nu mouse).

\section{Mini PDX drug sensitivity assays:}

BALB/c-nude mice(5-6 weeks) bearing Mini-PDX capsules were treated with drugs as detailed in Tables 1 for 7 days. Thereafter, the implanted capsules were removed, and tumor cell proliferation was evaluated using CellTiter Glo Luminescent Cell Viability Assay kit (G7571, Promega, Madison, WI, US) as instructed by the manufacturer. Luminescence was measured in terms of relative luminance unit (RFU) using a spectrophotometer (SpectraMax M3, Molecular Devices, Sunnyvale, CA, US). Tumor cell growth inhibition (TCGI) (\%) was calculated using the following formula:

TCGI\%=1-(Mean RLU of the treatment group on day 7-Mean RLU on day 0)/(Mean RLU of the vehicle group on day 7-Mean RLU on day 0$) \times 100 \%$

Each experiment was done in sextuplicate and mean values were reported. A positive drug response was considered present if TCGI was $\geq 45 \%(P<0.05)$, and a negative drug response was considered if TCGI was

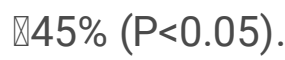

\section{Cell cycle analysis:}

After the indicated treatments, the cells were resuspended in PBS and fixed in $70 \%$ ethanol-PBS for 30 min at $4{ }^{\circ} \mathrm{C}$. Fixed cells were washed with PBS and treated with RNase at $37^{\circ} \mathrm{C}$ for 30 min. Finally, the cells were stained with propidium iodide $(\mathrm{PI})$ for $30 \mathrm{~min}$ at $37^{\circ} \mathrm{C}$. Samples were analyzed by flow cytometry using a FACSort cytometer (Becton-Dickinson, Franklin Lakes, NJ) and Cell Quest and ModFit software.

\section{RNAexpression data retrieval and analysis:}

Total RNA was extracted from cells using an RNA isolation kit (QIAGEN, Germany). GO enrichment and KEGG pathway analysis were implemented to discover pathways/processes in which the DEGs participated. The enriched functions of the genes were analyzed according to the $\mathrm{GO}$ terms of biological process (BP), cellular component (CC), and molecular function (MF). KEGG (http://www.genome.jp/KEGG/) is an online database that contains information on genomes, enzymatic pathways and biochemical. The DAVID (https://david.ncifcrf.gov/) was used to annotate and analyze the associated GO terms and KEGG pathways of the DEGs, with $p<0.05$ indicating significance.

\section{WES and Data processing:}

Genomic DNA was extracted from three PSTT cell lines with the DNeasy Kit (QIAGEN, Germany). Nextgeneration sequencing was conducted with the Illumina HiSeq X-TEN platform at CloudHealth Genomics. Reads were aligned to the human genome reference assembly (UCSC Genome Browser hg19) with the 
Burrows-Wheeler Aligner. Single-nucleotide variants (SNVs) and indels were called and analyzed by the Genome Analysis Toolkit. The SNVs with read depths less than 43 were filtered out. Then, we used ANNOVAR for functional annotation with OMIM, Gene Ontology, KEGG Pathway, SIFT, PolyPhen-2, MutationTaster, and the ExAC Browser.

\section{Results}

\section{Result 10Establishment and identification of three PSTT cell lines}

We established three PSTT cell lines derived from primary culture of surgically resected PSTT tissues from three PSTT patients. Immunofluorescence imaging showed that PSTT-1,2,3 cells all expressed HLAG, $\beta$-catenin, CD146, Muc4, hPL and hCG, which are typical molecular markers of intermediate cytotrophoblasts[Fig. 1A]. Then we observed the growth rate characteristics of the PSTT cell lines [fig. 1B]. We compared the homology of the PSTT cell lines and PSTT tumor tissue by short tandem repeat analysis [Fig. S1, Table 1]. Gene locus similarity between the PSTT cell lines and tumor tissues was as high as $100 \%$ (values greater than or equal to $80 \%$ are considered to indicate homologous samples). Moreover, the karyotype of the PSTT cell line was $46, X X$, the same as the normal female karyotype [fig. $1 \mathrm{C}]$.

\section{Result 2₫PSTT cell lines are resistant to MTX in vitro}

The preferred first-line therapy for PSTT is EP/EMA or EMA/EP. Therefore, we used the established cell lines to study chemosensitivity of the drugs in the EMA/EP regimen, including MTX, etoposide(VP-16), actinomycin $D(A c t-D)$, and cisplatin(DDP). In addition, we studied paclitaxel(TAX) from the TP/TE regimen and fluorouracil(5-FU) from the FA regimen. As shown in Fig. 2A, MTX had a significantly higher IC50 value in three PSTT cell lines $(4.922 \mathrm{mg} / \mathrm{ml}$ in PSTT-1, $4.525 \mathrm{mg} / \mathrm{ml}$ in PSTT-2, $5.117 \mathrm{mg} / \mathrm{ml}$ in PSTT-3) than in the choriocarcinoma JEG-3 $(0.0166 \mu \mathrm{g} / \mathrm{ml})$ and JAR cells $(0.01 \mu \mathrm{g} / \mathrm{ml})$. PSTT cell lines showed resistance to MTX, with a 50,000-fold increase in the IC50 value compared to that in choriocarcinoma cells. PSTT cell lines exposed to VP-16[fig. 2B], 5-FU[fig. 2C], TAX[fig. 2D], Act-D[fig. 2E], or DDP[fig. 2F] showed no significant difference in IC50 value compared to JAR and JEG-3 cells. In summary, PSTT cell lines show greater resistance to MTX than to six other common chemotherapy drugs. Therefore, we decided to focus on MTX resistance in PSTT cell lines.

\section{Result 3IPSTT cell line is resistant to MTX in vivo}

To verify the MTX resistance of PSTT-1 cells in vivo, we chose a new method-the mini xenograft(miniPDX) model. It is a new technology based on the traditional patient-derived xenograft (PDX), which has been patented (patent number: 201610918458.4). As shown in Fig. 3A, we loaded tumor cells in special Mini-PDX devices and then placed the devices subcutaneously in mice to simulate the in vivo tumor growth environment. Table 1 shows the dosage schedule for the four drugs. We weighed the mice during the drug administration period and found that Act-D significantly decreased body weight in both the PSTT-1 cohort and the JEG-3 cohort, while MTX had no significant effect on weight[fig. 3B]. MTX had a 
remarkable inhibitory effect in the JEG-3 cohort but no obvious effect in the PSTT-1 cohort [fig. 3B]. The relative growth rate showed that the PSTT-1 Mini-PDX model was resistant to MTX, Act-D, VP-16 and DDP compared to the JEG-3 xenograft model[fig. 3C]. In addition, we ascertained the relative luciferase units (RLUs) of cells derived from xenograft models after 7 days of drug administration, as this value represents tumor cell activity[fig. 3D]. The RLU results were consistent with the tumor volume results showing that PSTT-1 cells are resistant to these four drugs. Taken together, the in vitro and in vivo results indicate that PSTT-1 cells are consistently resistant to MTX.

\section{Result 4ロG1 phrase arrest and G1/S-phrase transition was dysregulated in PSTT cell lines}

Considering that MTX, a folate antagonist, is traditionally regarded as a proliferation inhibitor that blocks

the $S$ phase of the cell cycle ${ }^{(15)}$, we analyzed the cell cycle characteristics of the three PSTT cell lines. We found that the proportion of cells in S phase in the PSTT cell lines[fig. 4B] was significantly less than that in the JAR and JEG-3 cell lines[fig. 4A], suggesting that G1 phase arrested and the G1/S transition was dysregulated in PSTT cell lines compared to choriocarcinoma cell lines[fig. 4C]. As previously reported, cell cycle disorder is an important mechanism underlying the increased malignancy of tumor cells $s^{(22)}$, and alterations in the cell cycle have been considered responsible for drug resistance ${ }^{(23)}$. Therefore, we propose that loss of control of the G1/S transition may explain why PSTT is resistant to MTX in vivo and in vitro compared to MTX-sensitive choriocarcinoma cell lines.

\section{Result 5ロCell cycle associated DEGs were identified in PSTT cell lines}

To determine whether loss of control of the G1/S transition underlies the MTX resistance in PSTT, we analyzed RNA sequencing profiles of three PSTT cell lines and two choriocarcinoma cell lines. The results revealed 3,043 downregulated genes and 3,842 upregulated genes in PSTT cell lines[fig. 5A]. The heatmap[fig. 5B] of the DEGs were well separated between the MTX-resistant PSTT cell lines and the MTX-sensitive choriocarcinoma cell lines. Considering that a total of 6,885 DEGs were distinguished, we identified 16 gene clusters with different expression patterns across the PSTT and choriocarcinoma cell lines[fig. S2]. To determine the biological function of DEGs in PSTT, GO functional analysis was performed with a threshold of $p<0.05$. The $\mathrm{GO}$ enrichment analysis identified a total of $79 \mathrm{GO}$ categories[fig. S3]; different clusters were related to different biological processes, and clusters 13 and 16 were highly related to the cell cycle biological process[fig. 5C]. In addition, KEGG pathway analysis was performed to obtain more in-depth information of the biological functions of the DEGs in the PSTT cell lines. Clusters 13 and 16 were related to pathways involved in cell cycle, and cell cycle-associated genes accounted for the largest proportion in cluster 13[fig. 5D]. The expression levels of DEGs in PSTT cell lines and choriocarcinoma cell lines revealed that the cell cycle-associated DEGs in clusters 13 and 16 were all downregulated in PSTT cell lines [fig. 5E].

\section{Discussion}


PSTT is a rare form of $\operatorname{GTNs}^{(2,3,24)}$ and is less sensitive to conventional chemotherapy than choriocarcinoma. Hysterectomy is the most recognized treatment, which results in the loss of fertility ${ }^{(3-7)}$. Fertility-sparing therapy in PSTT patients is controversial because of the underlying chemoresistance. There are few reports on the basic mechanism of PSTT; the establishment of tumor cell lines plays an important role in basic tumor research, but there are currently no PSTT cell lines in authoritative cell banks. Thus far, there is only one report on the establishment of aPSTT cell line, IST-2 ${ }^{(25)}$. In contrast, we identified the expression of HLA-G, $\beta$-catenin, CD146, Muc4, hPL and hCG, which are more convicing markers of PSTT. The karyotype of IST-2 is 38-45, X,-X,-1,-2,-3,-6,-8,-22[cp11]/46,XX, and that of PSTT-1 is $46, X X$, which mimics the normal female karyotype. These karyotype results are consistent with previous study, which revealed the presence of an $X$ chromosome and the absence of $Y$ chromosome was observed in 20 PSTT tissues ${ }^{(26)}$. Besides, we compared the homology between the PSTT-1 cell line and PSTT tissue by STR analysis, further validating of the PSTT-1 cell line. Shih's study ${ }^{(25)}$ involved using IST2 to explore the role of MAPK pathway in PSTT invasion and migration rather than the chemoresistant mechanism. Therefore, our study firstly investigated the chemotherapy mechanism in PSTT.

The preferred first-line therapy is EP/EMA or EMA/EP in NCCN Guidelines. Therefore, we studied the chemosensitivity of PSTT to drugs in the EMA/EP regimen, including MTX, VP-16, Act-D, and DDP. In addition, we studied TAX from the TP/TE regimen and 5-FU from the FA regimen, which are also common regimens. In vitro, we found that PSTT were quite resistant to MTX, with a nearly 50,000-fold increase in the IC50 value, suggesting the methotrexate resistance of PSTT cell lines. Then, we verified the EMAEP regime in vivo because of MTX. We found that PSTT were resistant to the four drugs, while JEG-3 was still sensitive to the four drugs. Regarding the different results in vivo and in vitro, we considered that the drug effect might differ in vivo and in vitro because of the microenvironment, resulting in differences in physiological drug metabolism. In addition, these four drugs are clinically used as a combination regimen, and drug interactions play a role in vivo; therefore, a single drug may not work in vivo, and the four drugs might have no inhibitory effect on the Mini-PDX model when given separately, resulting in inconsistent drug effects in vitro and in vivo. The population of untreated JEG-3 cells generally increased almost 100-fold in 7 days, but that of PSTT cells increased no more than 2 fold, suggestive of the different growth rates of the two cell lines, which were also observed in vitro. In addition, PSTT cells could not form tumors by in situ or subcutaneous implantation, which may be also due to the slow growth.

MTX is the single most commonly used agent for the treatment of choriocarcinoma patients without metastases or with low risk of metastasis ${ }^{(12,13)}$, and approximately $30 \%$ of patients develop drugresistance for MTX monotherapy ${ }^{(14)}$. Without combining with other drugs to work, so it's relatively easy to study drug pharmacology and mechanism. All of the regimens for PSTT are combination therapies. The mechanisms of drug interactions are complicated, so it's difficult to perform drug effectiveness research, which may be why MTX-related studies in GTNs have mostly focused on drug resistance in choriocarcinoma. However, in our study, PSTT was least sensitive to MTX among the drugs in the commonly used combination chemotherapy regimen EMA/EP. JEG-3/MTX and JEG-3R cells have been used as resistance models $(27,28)$ to identify mechanism for chemotherapeutic resistance to MTX in 
choriocarcinoma ${ }^{(29)}$. JEG-3R showed more than 20-fold resistance compared to JEG-3/MTX. However, in our study, PSTT cell lines showed almost 50,000-fold resistance compared to JAR and JEG-3 cells. Therefore, we consider MTX resistance to be a unique characteristic of PSTT. We suspect that if patients have a poor response to EMA/EP, it is likely due to the MTX. Therefore, through our research, we can provide new directions for future drug research on PSTT.

We firstly began to explore the mechanism of PSTT in the field of MTX resistance. According to previous researches, the most studied mechanism of MTX resistance is the anti-folate metabolic pathway $(30,31)$. MTX tightly binds to dihydrofolate reductase(DHFR), blocking the reduction of dihydrofolate to tetrahydrofolic acid. ${ }^{(32)}$. Resistance to MTX may develop as a result of elevated DHFR activity or defective transport of methotrexate into malignant cells ${ }^{(32)}$. However, research assessing whether DHFR expression is correlated with MTX resistance in JEG-3 showed that DHFR gene transcript level correlated with MTX drug resistance at only a certain phase; thus, DHFR expression levels would be of limited use as a biomarker for the prediction of MTX chemoresistance ${ }^{(33)}$. In addition to DHFR, methylenetetrahydrofolate reductase (MTHFR) is also an important rate-limiting enzyme in folate metabolism. Studies have demonstrated that the MTHFR 677TT genotype in molar tissue is associated with ineffective MTX treatment in Japanese low-risk GTN patients, but there was no verification of the mechanism relating MTHFR function to MTX sensitivity ${ }^{(34)}$. Besides, Lssecka et al. found that neither the genotype for the 677CNT SNP or the 1298ANC SNP predict the therapeutic outcomes of women treated with single agent MTX for GTN, indicating that no correlation between MTHFR polymorphisms in molar tissue and MTX drug resistance ${ }^{(35)}$. As a result, DHFR- and MTHFR-associated studies have not clearly delineated the role of folate metabolism in MTX resistance in choriocarcinoma and the role of expression level of DHFR and MTHFR polymorphisms in prediction of MTX resistance is controversial for now. Therefore, our study did not focus on the folate metabolism pathway.

We found that G1 phrase arrest and dysregulation of G1/S-phrase transition in PSTT cell lines. Defective cell cycle events result in uncontrolled cell proliferation, which is considered as one of the hallmarks of cancer $^{(22)}$. Oncogenic processes exhibit their greatest effects by targeting $\mathrm{G} 1$ phase progression ${ }^{(15)}$ and alternations in cell cycle have also been considered responsible for drug resistance ${ }^{(23)}$. Many studies have verified that cell cycle disorders are related to MTX-resistant in some solid tumors ${ }^{(36,37)}$, therefore, we decided to focus our study of MTX-resistance on dysregulated cell cycle.

With the RNA sequencing profile, we found clusters of DEGs related to pathways involved in the cell cycle, suggesting that cell cycle-associated pathways are related to the MTX-resistance. Further verification of mechanism requires further study. We considered whether genetic mutation or epigenetic modification was responsible for these changes. We checked candidate genes of clusters 13 and 16 in the PSTT WGS dataset, and 27 genes in cluster 13[Supplementary Table2_cluster13] and 65 genes in cluster 16[Supplementary Table2_cluster16] were found in the WGS dataset. PSTT is a rare disease, so we excluded genetic variants with allele frequencies $\geq 0.0001$ according to the Exome Aggregation Consortium (ExAC) Browser and 1000 Genomes Project. In addition, the missense variants predicted to be 
potentially deleterious by SIFT, PolyPhen-2, and MutationTaster were kept for further evaluation. Through the above screening, we identified 10 genes in cluster 13 and 8 genes in cluster 16, and 6(MTBP, FBXO5, NEK2, POLE, MSH2, BLM) of the 18 genes function in the G1/S transition of mitosis, cell cycle regulation from $\mathrm{G} 1$ to $S$ phase, $S$ phase of mitosis, cell cycle arrest and other cell cycle-related functions [Supplementary Table 3_6 genes]. Mutations of these 6 genes may account for the dysregulation of the cell cycle in PSTT and might be responsible for MTX resistance in PSTT cell lines. The other DEGs that were not found in WGS dataset might be the downstream of the 6 genes, this must be further investigated through more in-depth mechanism studies. The limitations of our study are that we didn't further verified the specific pathways and didn't identified the function of the 6 genes in PSTT.

In conclusion, we successfully established PSTT cell lines and generated an in vitro experimental model for PSTT research. We found that PSTT is extremely resistant in vitro and in vivo to MTX, a component of the common clinical combination regime, EMAEP. Exploration of the MTX resistance mechanism focused on dysregulation of G1/S transition and cell cycle arrest. The RNA sequencing profile, along with the WGS data for PSTT, verified the cell cycle pathway as the potential mechanism of resistance. We found 6 potential cell cycle-associated gene mutations that might account for the MTX resistance in PSTT.

\section{List Of Abbreviations}

PSTT: Placenta site trophoblastic tumor; HLA-G: human leucocyte antigen-G; hPL: human placental lactogen; hCG: human chorionic gonadotropin; MTX: methotrexate; VP-16: etoposide; Act-D: actinomycinD; DDP: cisplatin; 5-FU: fluorouracil; TAX: paclitaxel; Mini PDX: Mini patient-derived xenograft; WGS: Whole genome sequencing.

\section{Declarations}

Ethics approval: The present study was approved by the ethical committee of the Obstetrics and Gynecology Hospital of Fudan University (IRB number:2016-56). The animal experiments was also approved by the animal ethical committee.

Consent for publication: Not applicable.

Availability of data and materials: The analyzed data sets generated during the present study are available from the corresponding author on reasonable request.

Competing interests: The authors declare that they have no competing interests.

Funding: This work was supported by National Key R\&D Program of China(2016YFC1303100), Shanghai Science and Technology Commission(18140902502 and 18411963300), Shanghai Municipal Health and Family Planning Commission (2018BR26 and ZYKC201701020), Shanghai Shenkang Hospital Development Center(SHDC12018X13). 


\section{Authors' contributions:}

The conception and design of study: Yu Kang and Congjian Xu;

The acquisition of data: Jing Xu, Ling Zhang, Qiyu Liu;

Analysis data: Jing Xu, Ling Zhang, Luyao Ren, Baozhu Cai, Ke Li, Yunke Huang, Jing Wang, Qin Li;

Drafting the article: Jing Xu;

Revising it critically for important intellectual content: Jing Xu, Yu Kang, Congjian Xu;

Final approval of the version: Yu Kang and Congjian Xu.

Acknowledgements: We thank Feng Zhang Lab(School of Life Sciences, Fudan University) for their support.

\section{References}

1. Scully RE, Young RH. Trophoblastic pseudotumor: a reappraisal. The American journal of surgical pathology. 1981;5(1):75-6.

2. Hassadia A, Gillespie A, Tidy J, Everard RGNJ, Wells M, Coleman R, et al. Placental site trophoblastic tumour: clinical features and management. Gynecologic oncology. 2005;99(3):603-7.

3. Schmid P, Nagai Y, Agarwal R, Hancock B, Savage PM, Sebire NJ, et al. Prognostic markers and longterm outcome of placental-site trophoblastic tumours: a retrospective observational study. Lancet. 2009;374(9683):48-55.

4. Ngan HYS, Seckl MJ, Berkowitz RS, Xiang Y, Golfier F, Sekharan PK, et al. Update on the diagnosis and management of gestational trophoblastic disease. International journal of gynaecology and obstetrics: the official organ of the International Federation of Gynaecology and Obstetrics. 2018;143 Suppl 2:79-85.

5. Horowitz NS, Goldstein DP, Berkowitz RS. Placental site trophoblastic tumors and epithelioid trophoblastic tumors: Biology, natural history, and treatment modalities. Gynecologic oncology. 2017;144(1):208-14.

6. Shen X, Xiang Y, Guo L, Feng F, Wan X, Xiao Y, et al. Fertility-preserving treatment in young patients with placental site trophoblastic tumors. International journal of gynecological cancer : official journal of the International Gynecological Cancer Society. 2012;22(5):869-74.

7. Saso S, Haddad J, Ellis P, Lindsay I, Sebire NJ, Mclndoe A, et al. Placental site trophoblastic tumours and the concept of fertility preservation. BJOG. 2012;119(3):369-74; discussion 74.

8. Raza A, Ahmad Z, Muzzaffar S. Placental site trophoblastic tumor (PSTT) with metastases to lungs and adrenal glands. Journal of the College of Physicians and Surgeons-Pakistan : JCPSP. 2006;16(2):150-1. 
9. Ohmaru T, Yamakawa H, Netsu S, Nokubi M, Konno R. Placental site trophoblastic tumor (PSTT) with multiple metastases and extremely poor prognosis. International journal of clinical oncology. 2009;14(5):452-6.

10. Behtash N, Karimi Zarchi M. Placental site trophoblastic tumor. Journal of cancer research and clinical oncology. 2008;134(1):1-6.

11. Bolze PA, Mathe M, Hajri T, You B, Dabi Y, Schott AM, et al. First-line hysterectomy for women with low-risk non-metastatic gestational trophoblastic neoplasia no longer wishing to conceive. Gynecologic oncology. 2018;150(2):282-7.

12. Abrao RA, de Andrade JM, Tiezzi DG, Marana HR, Candido dos Reis FJ, Clagnan WS. Treatment for low-risk gestational trophoblastic disease: comparison of single-agent methotrexate, dactinomycin and combination regimens. Gynecologic oncology. 2008;108(1):149-53.

13. Stevens FT, Katzorke N, Tempfer C, Kreimer U, Bizjak GI, Fleisch MC, et al. Gestational Trophoblastic Disorders: An Update in 2015. Geburtshilfe und Frauenheilkunde. 2015;75(10):1043-50.

14. Biscaro A, Braga A, Berkowitz RS. Diagnosis, classification and treatment of gestational trophoblastic neoplasia. Rev Bras Ginecol Obstet. 2015;37(1):42-51.

15. Sherr CJ. Cancer cell cycles. Science. 1996;274(5293):1672-7.

16. Wu HB, Lv WF, Wang YX, Li YY, Guo W. BCL6 promotes the methotrexate-resistance by upregulating ZEB1 expression in children with acute B lymphocytic leukemia. Eur Rev Med Pharmacol Sci. 2018;22(16):5240-7.

17. Chen Y, Bian L, Zhang Y. MiR-505 mediates methotrexate resistance in colorectal cancer by targeting RASSF8. J Pharm Pharmacol. 2018;70(7):937-51.

18. Monga M, Sausville EA. Developmental therapeutics program at the $\mathrm{NCl}$ : molecular target and drug discovery process. Leukemia. 2002;16(4):520-6.

19. Lee $\mathrm{KH}$, Rhee $\mathrm{KH}$. Correlative effect between in vivo hollow fiber assay and xenografts assay in drug screening. Cancer Res Treat. 2005;37(3):196-200.

20. Suggitt M, Swaine DJ, Pettit GR, Bibby MC. Characterization of the hollow fiber assay for the determination of microtubule disruption in vivo. Clinical cancer research : an official journal of the American Association for Cancer Research. 2004;10(19):6677-85.

21. Phillips RM, Pearce J, Loadman PM, Bibby MC, Cooper PA, Swaine DJ, et al. Angiogenesis in the hollow fiber tumor model influences drug delivery to tumor cells: implications for anticancer drug screening programs. Cancer research. 1998;58(23):5263-6.

22. Zhang Y, Chen S, Wei C, Rankin GO, Ye X, Chen YC. Flavonoids from Chinese bayberry leaves induced apoptosis and G1 cell cycle arrest via Erk pathway in ovarian cancer cells. Eur J Med Chem. 2018;147:218-26.

23. Yano S, Takehara K, Tazawa H, Kishimoto H, Urata Y, Kagawa S, et al. Cell-cycle-dependent drugresistant quiescent cancer cells induce tumor angiogenesis after chemotherapy as visualized by realtime FUCCl imaging. Cell cycle. 2017;16(5):406-14. 
24. Hyman DM, Bakios L, Gualtiere G, Carr C, Grisham RN, Makker V, et al. Placental site trophoblastic tumor: analysis of presentation, treatment, and outcome. Gynecologic oncology. 2013;129(1):58-62.

25. Kobel M, Pohl G, Schmitt WD, Hauptmann S, Wang TL, Shih le M. Activation of mitogen-activated protein kinase is required for migration and invasion of placental site trophoblastic tumor. The American journal of pathology. 2005;167(3):879-85.

26. Hui P, Wang HL, Chu P, Yang B, Huang J, Baergen RN, et al. Absence of $Y$ chromosome in human placental site trophoblastic tumor. Modern pathology : an official journal of the United States and Canadian Academy of Pathology, Inc. 2007;20(10):1055-60.

27. Zhao J, Xiang Y, Xiao C, Guo P, Wang D, Liu Y, et al. AKR1C3 overexpression mediates methotrexate resistance in choriocarcinoma cells. International journal of medical sciences. 2014;11(11):1089-97.

28. Peng Z, Zhang C, Zhou W, Wu C, Zhang Y. The STAT3/NFIL3 signaling axis-mediated chemotherapy resistance is reversed by Raddeanin A via inducing apoptosis in choriocarcinoma cells. Journal of cellular physiology. 2018;233(7):5370-82.

29. Snow K, Judd W. Characterisation of adriamycin- and amsacrine-resistant human leukaemic T cell lines. British journal of cancer. 1991;63(1):17-28.

30. Fotoohi AK, Assaraf YG, Moshfegh A, Hashemi J, Jansen G, Peters GJ, et al. Gene expression profiling of leukemia T-cells resistant to methotrexate and 7-hydroxymethotrexate reveals alterations that preserve intracellular levels of folate and nucleotide biosynthesis. Biochem Pharmacol. 2009;77(8):1410-7.

31. Kager L, Cheok M, Yang W, Zaza G, Cheng Q, Panetta JC, et al. Folate pathway gene expression differs in subtypes of acute lymphoblastic leukemia and influences methotrexate pharmacodynamics. J Clin Invest. 2005;115(1):110-7.

32. Isonishi S, Terashima Y. [Methotrexate in gynecologic oncology]. Gan to kagaku ryoho Cancer \& chemotherapy. 1996;23(14):1896-900.

33. Han $B$, Xiang $Y$, Wang $Y$, Wang $Z$, Zhang $H$, Huang S. Dihydrofolate reductase transcript level is not suitable for methotrexate-resistance prediction in choriocarcinoma cell line. International journal of gynecological cancer : official journal of the International Gynecological Cancer Society. 2010;20(7):1259-63.

34. Qu J, Usui H, Kaku H, Shozu M. Presence of the methylenetetrahydrofolate reductase gene polymorphism MTHFR C677T in molar tissue but not maternal blood predicts failure of methotrexate treatment for low-risk gestational trophoblastic neoplasia. Eur J Pharmacol. 2017;794:85-91.

35. Lasecka L, Dixon PH, Molokhia M, Sharma N, Schleh A, Wang CM, et al. 667C>T and 1298A>C polymorphisms of MTHFR do not predict response to methotrexate in patients with gestational trophoblastic neoplasia. Gynecologic oncology. 2011;123(3):605-9.

36. Wu KF, Liang WC, Feng L, Pang JX, Waye MM, Zhang JF, et al. H19 mediates methotrexate resistance in colorectal cancer through activating Wnt/beta-catenin pathway. Experimental cell research. 2017;350(2):312-7. 
37. Longo-Sorbello GS, Bertino JR. Current understanding of methotrexate pharmacology and efficacy in acute leukemias. Use of newer antifolates in clinical trials. Haematologica. 2001;86(2):121-7.

\section{Table}

\begin{tabular}{|c|c|c|}
\hline \multicolumn{3}{|c|}{ Table 1 Drug Administration } \\
\hline MTX & $6 \mathrm{mg} / \mathrm{kg}$ & IP, QD*5 \\
\hline Act-D & $0.1 \mathrm{mg} / \mathrm{kg}$ & IP, QD*5 \\
\hline VP-16 & $20 \mathrm{mg} / \mathrm{kg}$ & IP, Q4D*2 \\
\hline DDP & $5 \mathrm{mg} / \mathrm{kg}$ & IP, Q4D*2 \\
\hline
\end{tabular}

Figures 
A Negative Control HLA-G

$\beta$-catenin

CD146

hPL

hCG

PSTT-1
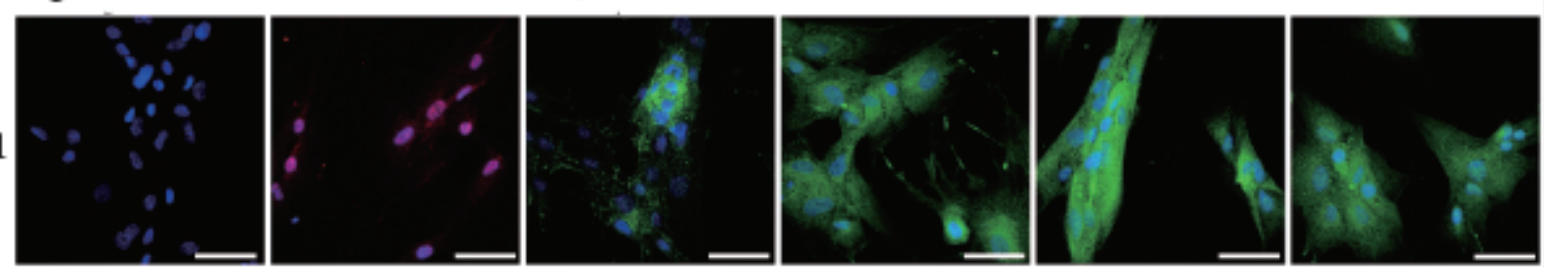

PSTT-2
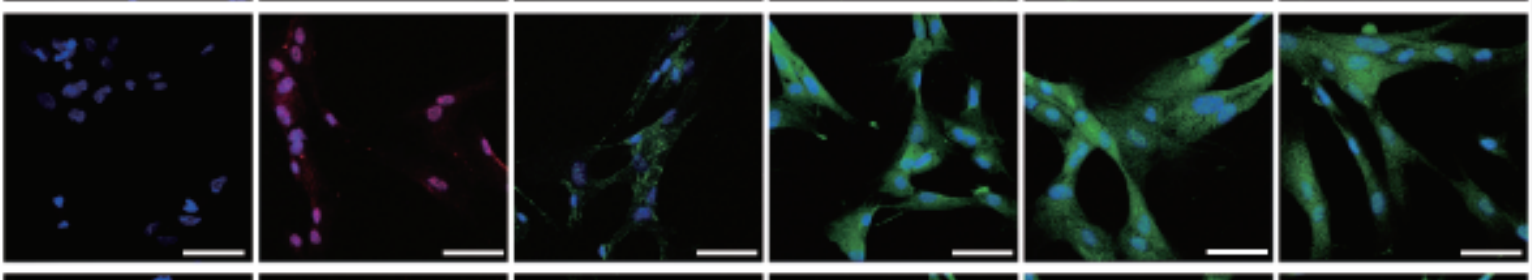

PSTT-3
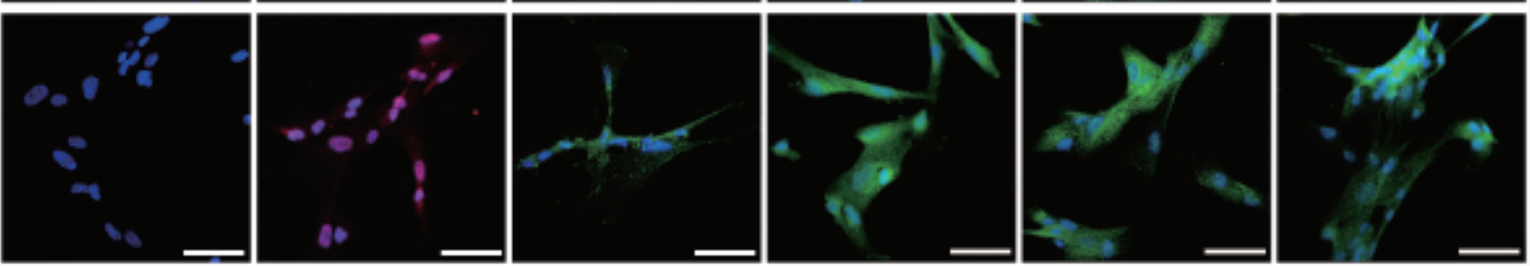

B

C
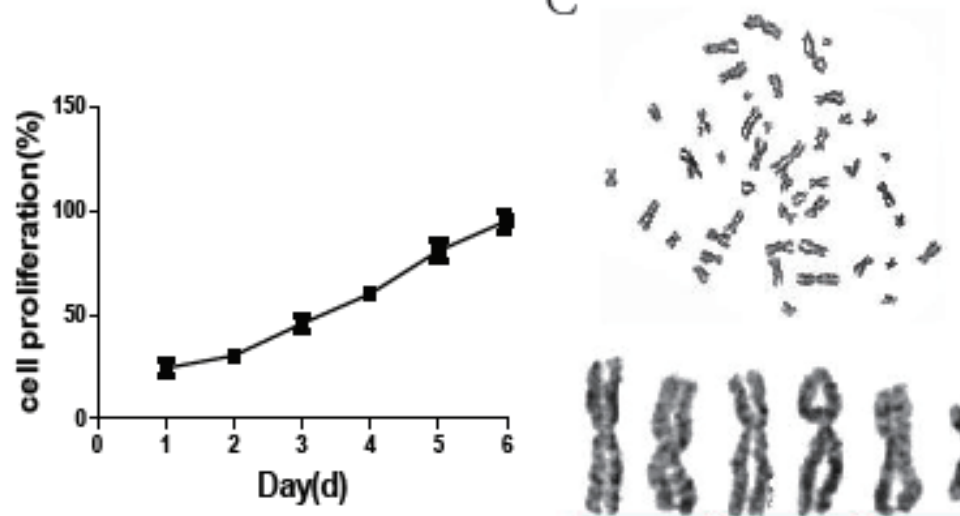

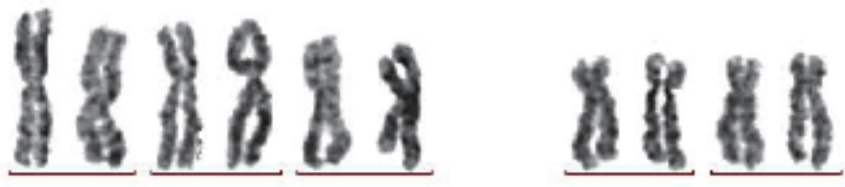

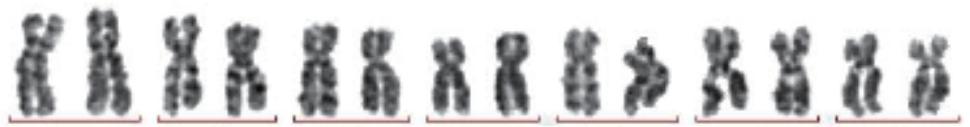

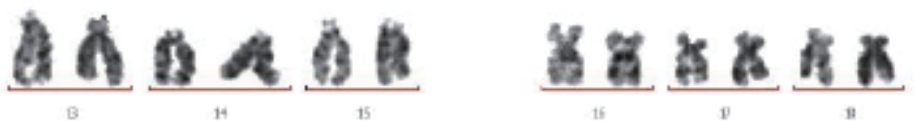

6rox sen

\section{Figure 1}

Establishment and identification of PSTT-1, PSTT-2 and PSTT-3 cell lines. A) Expression of HLA-G, $\beta$ catenin, CD146, Muc4, hPL and hCG in three PSTT cell lines, scale bars, $250 \mu \mathrm{m}$. B) Growth curve of PSTT cell line. C) Chromosomal karyotype analysis of PSTT cell line. 


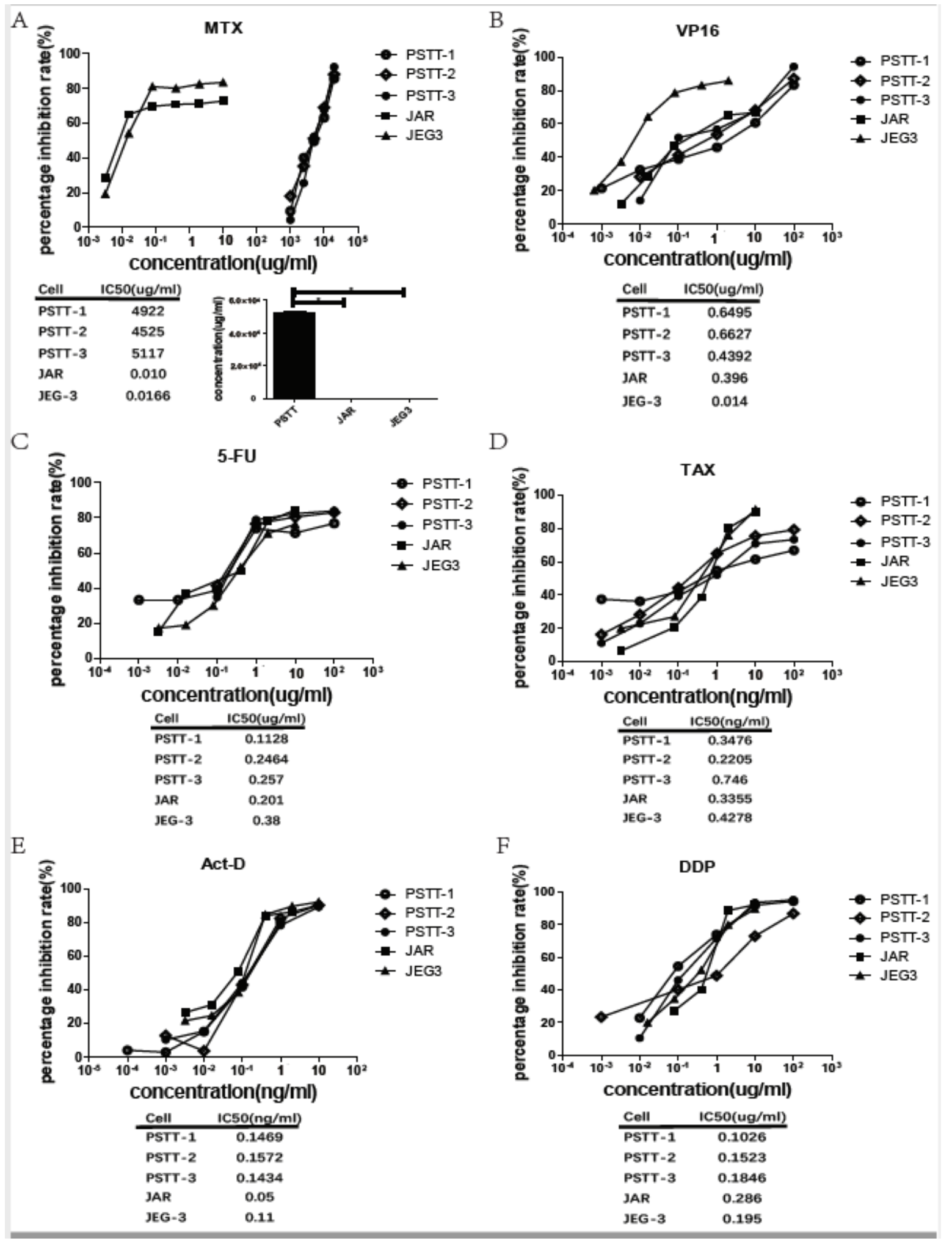

Figure 2

PSTT cell lines are resistant to MTX in vitro. A) IC50 dose response curves of methotrexate in PSTT-1,2,3 cells vs. JEG-3 cells and JAR cells. B) IC50 dose response curves of etoposide in PSTT-1,2,3 cells vs. JEG3 cells and JAR cells. C) IC50 dose response curves of 5-Fluorouracil in PSTT-1,2,3 cells vs. JEG-3 cells and JAR cells. D) IC50 dose response curves of taxol in PSTT-1,2,3 cells vs. JEG-3 cells and JAR cells. E)IC50 dose response curves of actinomycin D in PSTT-1,2,3 cells vs. JEG-3 cells and JAR cells. F) IC50 
dose response curves of Cisplatin in PSTT-1,2,3 cells vs. JEG-3 cells and JAR cells. Data represent mean $\pm S D$ from at least three independent experiments, ${ }^{*} p<0.001$.

A

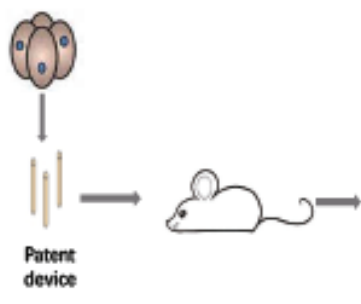

B

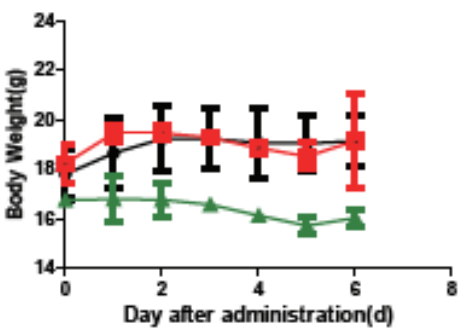

JEG-3

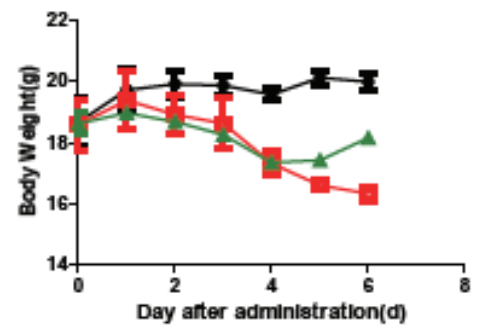

D
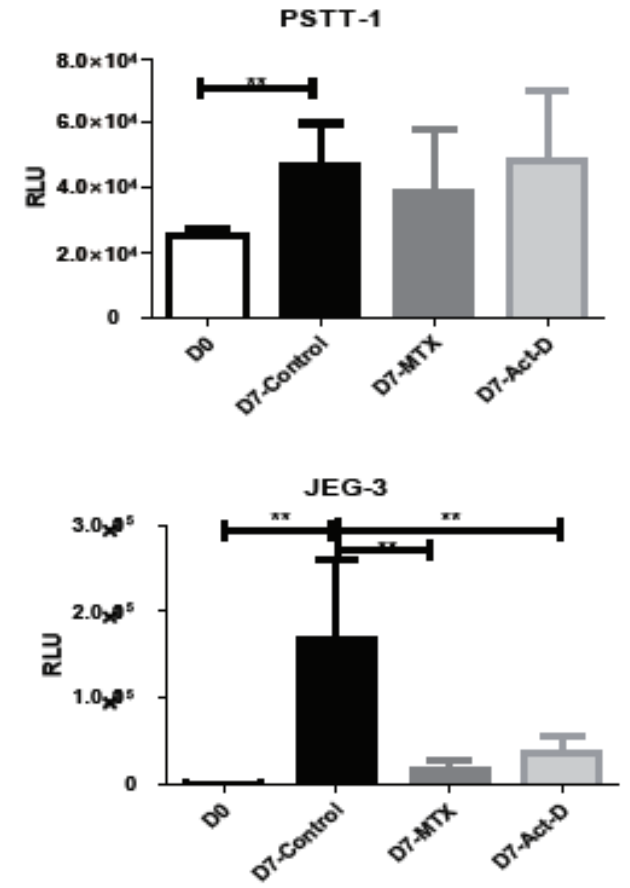

$\mathrm{C}$

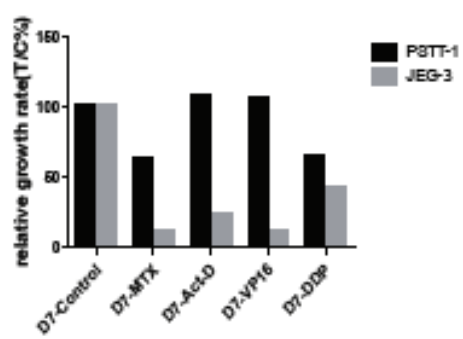

PSTT-1
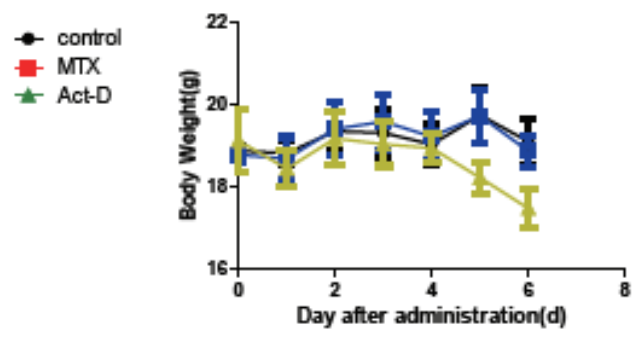

JEG-3
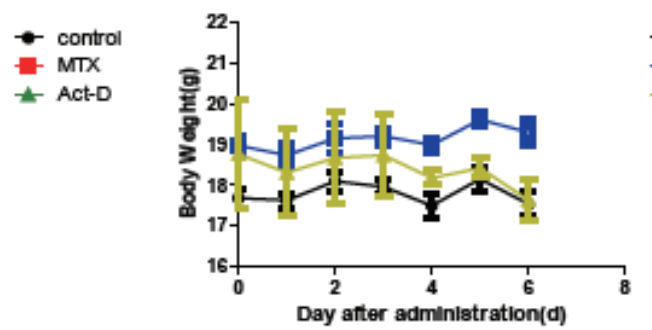

Dontro
$\rightarrow-$ VP-16
- DDP

Dontro
- col
- VP-16
- DDP
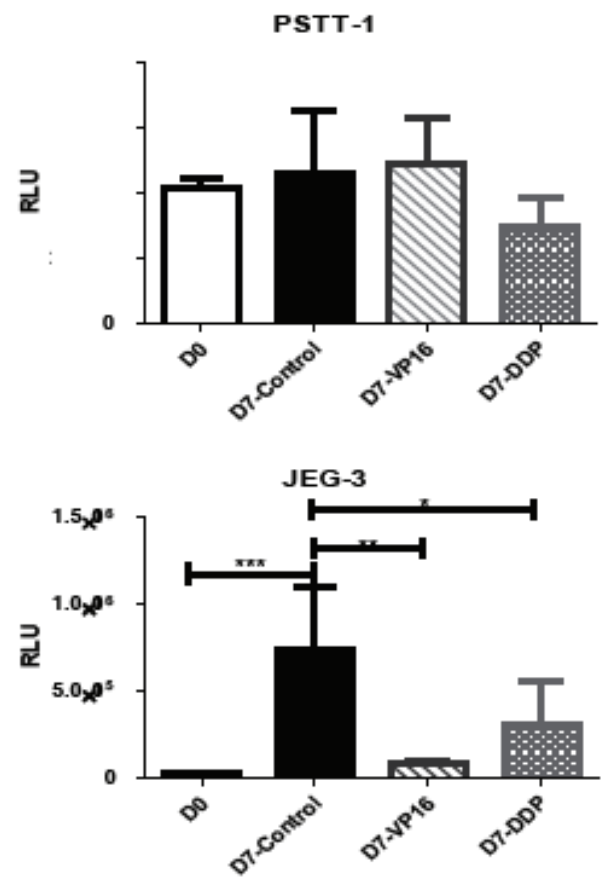

Figure 3

PSTT is resistant to MTX in vivo. A) Process of mini-PDX models establishment. B) Body weight change curve of mice during the drug administration period. C) The relative tumor growth rate in the drug-treated groups and the control group. " $T$ " and "C" represent the average tumor volume (TV) in the drug-treated 
group and the control group, respectively. D) Relative luciferase units (RLUs) of cells derived from xenograft models after 7 days of drug administration. The RLU represents tumor cell activity. Data are presented as the mean \pm SD of at least three independent experiments, ${ }^{* \star *} p<0.001,{ }^{* \star} p<0.01,{ }^{*} p<0.05$.

A

B
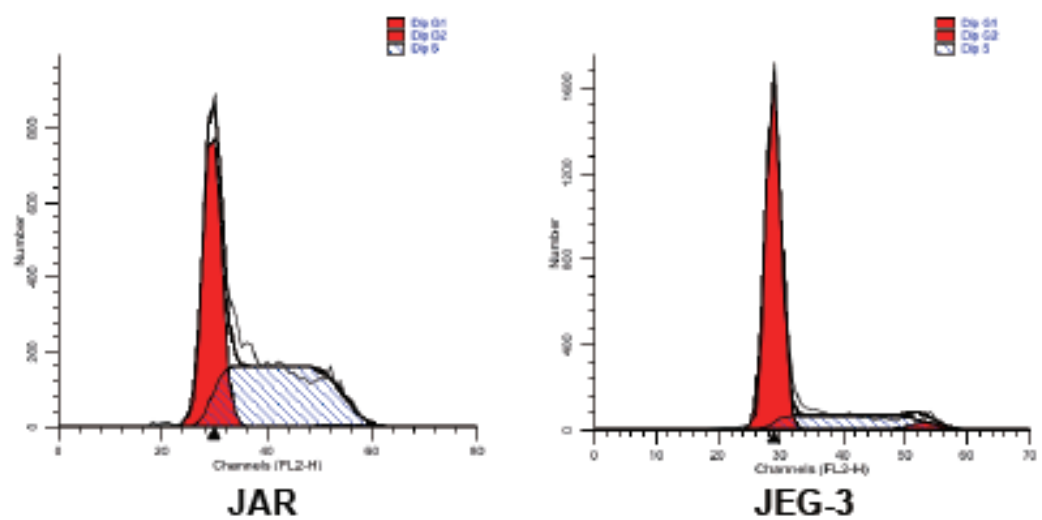

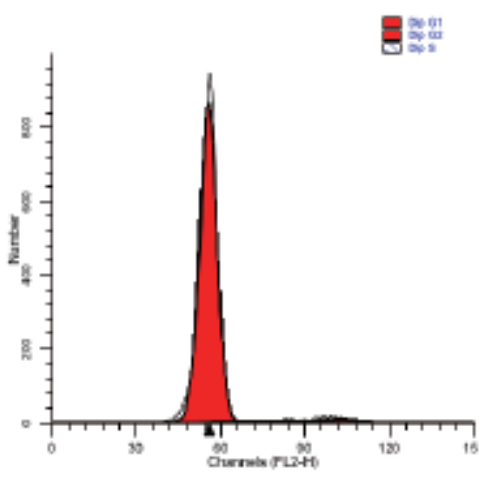

PSTT-1

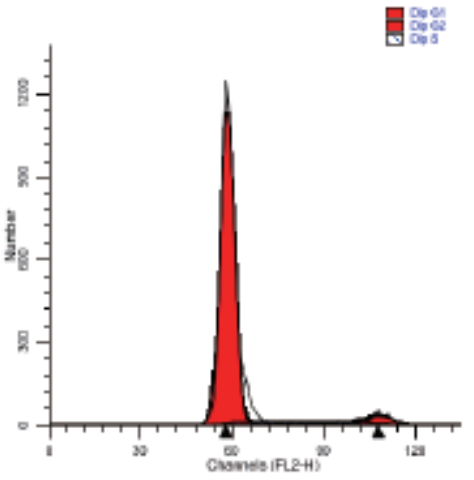

PSTT-2

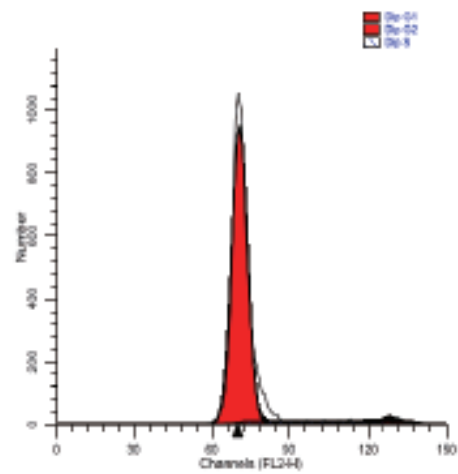

PSTT-3

C

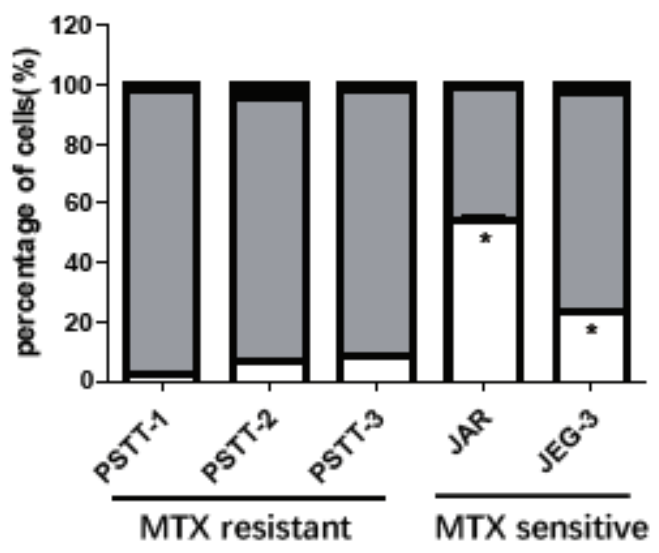

Figure 4

G1 phrase arrest and G1/S-phrase transition was dysregulated in three PSTT cell lines. A) Cell cycle analysis of choriocarcinoma JEG-3 and JAR cells. There independent experiments were performed. B) Cell cycle analysis of PSTT-1, PSTT-2, PSTT-3 cells. There independent experiments were performed. C) The percentages of cells in different phrase are expressed as the mean $\pm S D, * P \llbracket 0.001$. 


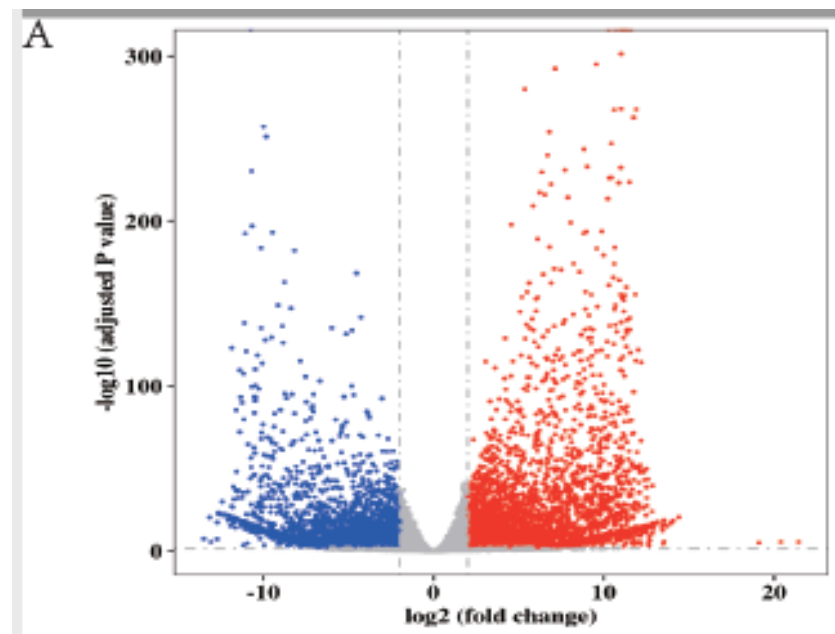

C

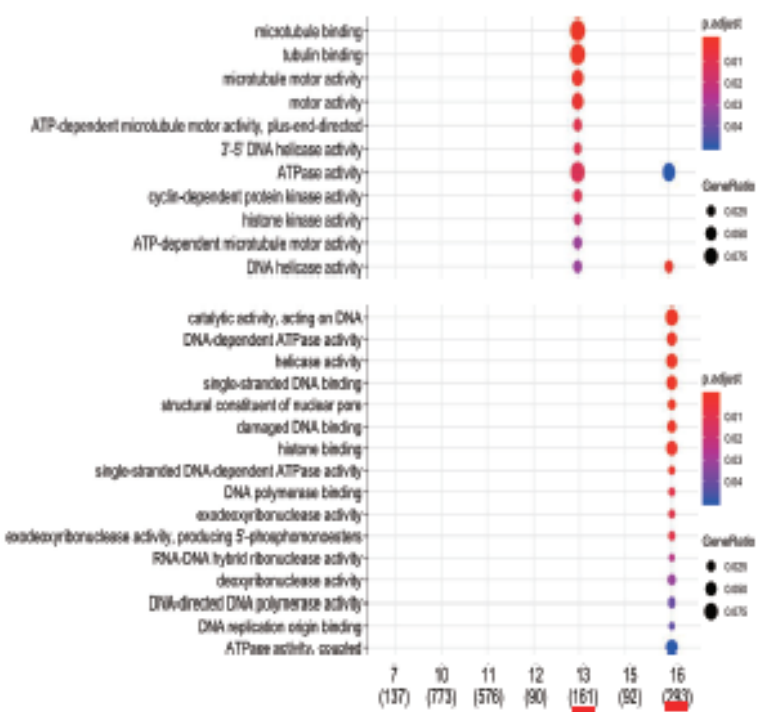

$\mathrm{E}$
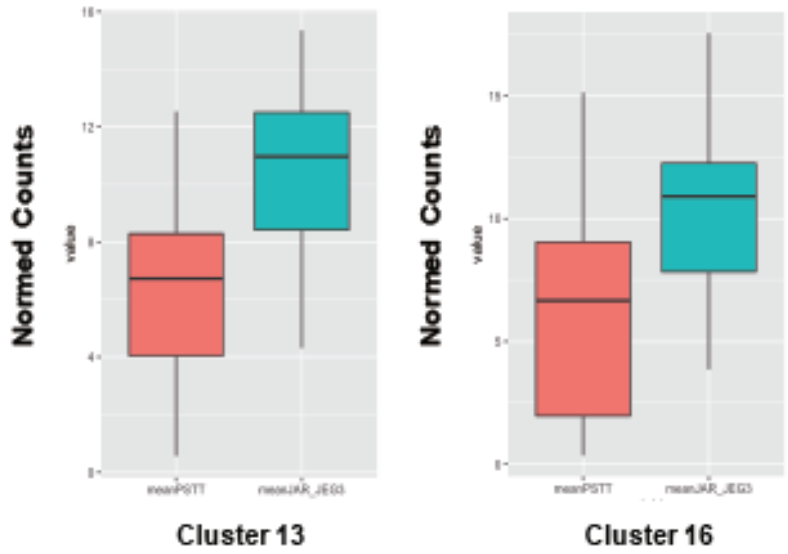

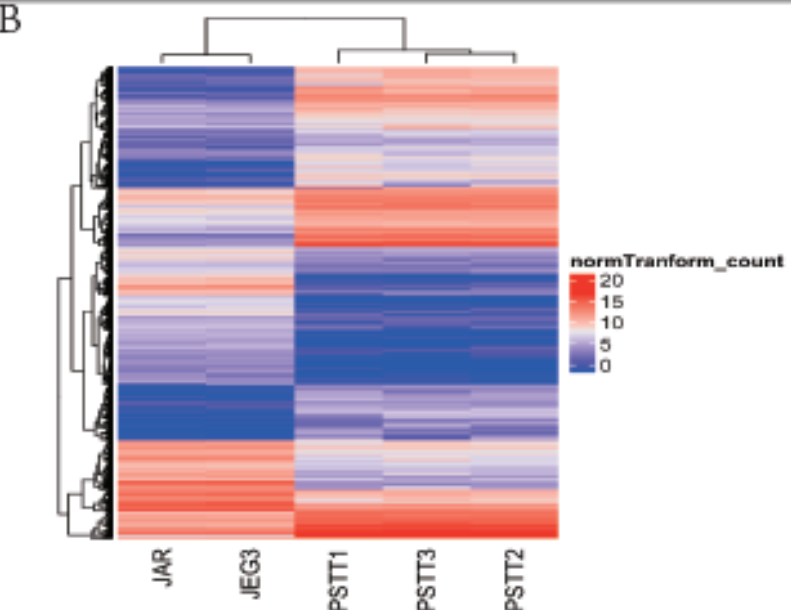

$\mathrm{D}$

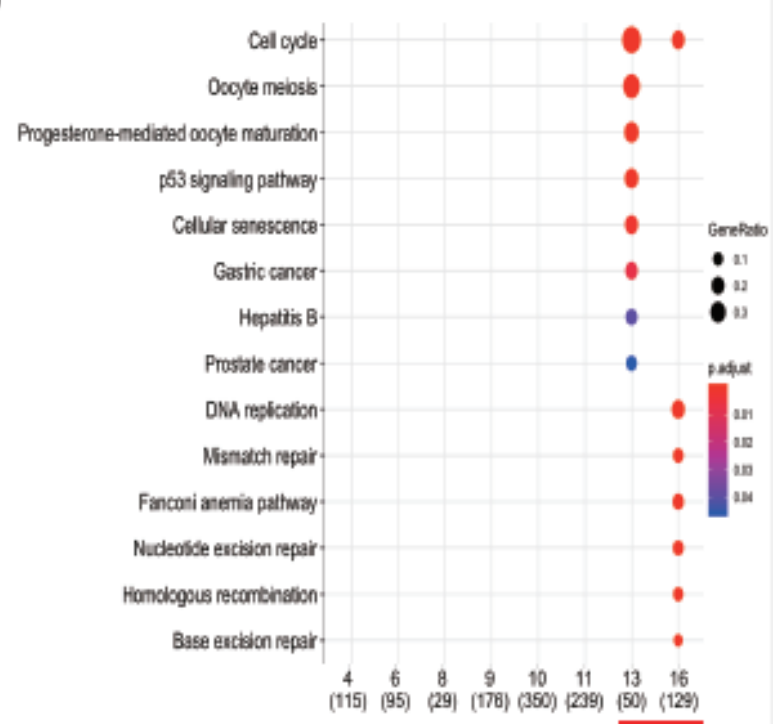

\section{Figure 5}

Cell cycle associated DEGs were identified in three PSTT cell lines. A) Volcano plot of differentially expressed genes of PSTT cell lines and choriocarcinoma cell lines. (Threshold: Raw count sum of 5 samples $>10$, Adjusted $p$ value $<0.01$, Absolute log2Fold Change $>2$.) B) Heatmap of DEGs between PSTT cell lines and choriocarcinoma cell lines. C) Gene ontology enrichment analysis of the differentially 
expressed genes. D) Kyoto Encyclopedia of Genes and Genomes pathway analysis of the differentially expressed genes. E) Expression level of DEGs in PSTT cell lines and choriocarcinoma cell lines.

\section{Supplementary Files}

This is a list of supplementary files associated with this preprint. Click to download.

- supplementarytable2cluster13.xlsx

- supplementaryFigure3.png

- supplementarytable2cluster16.xlsx

- supplementaryFigure1.tif

- supplementaryFigure4.png

- supplementaryFigure2.tif

- supplementarytable36genes.xlsx

- supplementarytable1STR.xlsx 